\title{
SEMBRANDO SOBERANÍA ALIMENTARIA PARA OTROS MODELOS DE VIDA EN EUSKAL HERRIA. LAS POLÍTICAS PÚBLICAS LOCALES COMO HERRAMIENTA DE TRANSFORMACIÓN ${ }^{1}$
}

\author{
Silvia Piris \\ Militante de Bizilur e integrante del Grupo de Investigación sobre Cooperación Crítica \\ y Movimientos Sociales del Instituto Hegoa (UPV/EHU)
}

DOI: $10.1387 /$ lan-harremanak.16102

\section{ABSTRACT}

En el contexto actual de crisis sistémica del modelo capitalista la revisión critica de cómo se está entendiendo la agricultura y la producción de alimentos resulta central. El modelo agroindustrial capitalista, basado en ese principio de entender la alimentación como mercancía y no como derecho, y estructurado en torno a los intereses de las grandes empresas transnacionales, genera vulnerabilidad alimentaria, ecoló-

${ }^{1}$ El presente artículo está basado en el documento Sembrando Soberanías para otros modelos de vida en Euskal Herria. Algunas propuestas para la construcción de politicas públicas desde la soberanía alimentaria (junio de 2015), disponible en: http://www.elikaherria.eus/documento-de-politicaspublicas-locales-y-soberania-alimentaria-en-euskal-herria/. Sembrando soberanías nace de la colaboración y alianza entre las organizaciones Bizilur, Etxalde y EHNE Bizkaia, con el objetivo de aportar claves y fortalecer las capacidades de los municipios de Euskal Herria para impulsar la soberanía alimentaria en el ámbito de las políticas públicas locales. Para ello, en esta publicación, además de desarrollar un marco teórico que pueda ser útil para abordar la construcción de estas políticas, se recogen diferentes líneas de actuación y experiencias, algunas de ellas ya en marcha, y que nos pueden permitir vislumbrar posibles caminos. Este artículo recoge y resume las ideas centrales presentadas en dicha publicación y es por tanto, fruto del trabajo colectivo de las organizaciones anteriormente mencionadas. 
gica, social y económica. Frente a esto, la agenda de la soberanía alimentaria, como enfoque integral, abierto y alternativo, se nos presenta como una propuesta contrahegemónica de primer orden, los territorios como ámbito estratégico para la implementación de esta agenda, y las politicas públicas como herramienta tractora fundamental para transitar hacia otros modelos de organización sociales, politicos y económicos que coloquen la alimentación y la sostenibilidad de la vida en el centro.

Palabras clave: soberania alimentaria, politicas públicas, territorio, transformación.

In the current context of a systemic crisis of capitalism a critical review of how agriculture and food production are being seen is of central importance. The capitalist agro-industrial model based on the principle of seeing food as merchandise rather than as a right, and organised around the interests of the big transnational corporations, leads to food, ecological, social and economic vulnerability. In the face of this, the food sovereignty agenda, as a holistic, open and alternative approach, represents an anti-hegemonic proposition of the first order: regions as the strategic sphere for implementing this agenda, and public policy as the fundamental tool to drive the transition to other models of social, political and economic organisation that place food and the sustainability of life at centre stage.

Keywords: food sovereignty, public policy, territory, transformation.

Sistema kapitalistaren krisi sistemiko egoera honetan, funtsezkoa da nekazaritzaren eta elikadura ekoizpenaren ulermenaren berrikusketa kritikoa egitea. Nekazaritza eta industria modelo kapitalistak _ elikadura merkantzia gisa ulertzen duena eta ez eskubide moduan, eta enpresa transnazional handien interesen inguruan egituratzen dena- elikagaien zaurgarritasuna, zaurgarritasun ekologikoa, soziala eta ekonomikoa sortzen ditu. Horren aurrean, elikadura-burujabetzaren agenda oinarrizko kontra-hegemonia proposamen gisa aurkezten da; lurraldeak agenda hori ezartzeko jardun-eremu gisa; eta politika publikoak oinarrizko trakzio-tresna gisa, elikadura eta bizitzaren jasangarritasuna ardatz moduan dituzten antolakuntza modelo sozialetara, politikoetara eta ekonomikoetara igarotzeko.

Hitz gakoak: elikadura-burujabetza, politika publikoak, lurraldea, eraldaketa. 


\section{Introducción}

En las siguientes líneas vamos a tratar de presentar algunas ideas que nos permitan entender en qué medida el marco teórico y la agenda de la soberanía alimentaria, como un enfoque político integral, abierto y alternativo, puede y debe ser una propuesta de primer orden en ese disputar la centralidad de las grandes corporaciones trasnacionales que nos convoca en la presente publicación. Y más concretamente, vamos a tratar de exponer la potencialidad de esta propuesta desde una de las herramientas posibles, evidentemente no la única, para su desarrollo: las políticas públicas locales. Así estructuramos las siguientes páginas intentando dar respuesta a dos grandes cuestiones. En primer lugar, nos centraremos en exponer por qué consideramos que la soberanía alimentaria presenta una alternativa real al poder de las empresas transnacionales y a la privatización de la vida. Para ello, abordaremos cuál es el diagnóstico de la realidad de la que partimos y algunas de las que consideramos son líneas centrales de la agenda por la soberanía alimentaria. En un segundo momento, trataremos la cuestión específica de las políticas públicas, como herramienta que acompañe la transformación en nuestros territorios. Nos centraremos en presentar, por un lado, la mirada desde la cual proponemos abordar el aterrizaje de la agenda de la soberanía alimentaria en el ámbito local, enfoque que nombramos como intersección de soberanias para, en segundo lugar, aportar algunas claves que explican desde qué mirada a lo público (y a las políticas públicas) realizamos esta propuesta.

\section{Soberanía alimentaria como propuesta alternativa frente al poder de las transnacionales y a la privatización}

Abordamos en este primer punto los que consideramos son elementos centrales para aproximarnos al marco y a la agenda de la soberanía alimentaria como propuesta alternativa al poder de las empresas transnacionales. Para ello, expondremos en primer lugar algunas pinceladas de la mirada a la realidad de la que partimos, para presentar, en un segundo momento, las claves que consideramos fundamentales para comprender el potencial transformador de esta agenda. 


\subsection{Crisis sistémica y modelo agroindustrial: desde dónde miramos el mundo}

Comenzamos por presentar algunos elementos centrales para ubicar la mirada al mundo y, concretamente, al mundo campesino y a la alimentación, que podemos decir articulan la agenda de la soberanía alimentaria. Desde esta propuesta se entiende que nos encontramos en un momento de crisis profunda, momento que nos coloca ante debates sobre qué sociedad, qué formas de producción, qué trabajos, qué instituciones, qué políticas públicas, etc., queremos y necesitamos, para hacer frente a las dinámicas actuales de privatización y profundización de las desigualdades, soñar y construir alternativas que nos permitan avanzar en términos de justicia, bienestar y sostenibilidad.

Así, nos unimos a las diversas autoras/es que definen la actual crisis como civilizatoria, es decir, que apuntan a que lo que realmente está en crisis son los valores del sistema capitalista (Fernández, Piris y Ramiro, 2013) ${ }^{2}$. Por tanto, entendemos que nos encontramos en un momento de quiebra de un sistema que, a partir de su necesidad constante de maximización del beneficio de sus élites, y con los diferentes sistemas de opresión en los que se apuntala (productivismo, patriarcado, colonialismo, democracia de baja intensidad...) está generando desigualdad, insostenibilidad e ingobernabilidad. Un sistema que es incapaz de garantizar un mínimo bienestar a las grandes mayorías sociales, un sistema que está impidiendo la reproducción de la vida priorizando las lógicas depredadoras del capital (Fernández, 2015) ${ }^{3}$.

2 En este sentido, afirman que nos encontramos ante una crisis civilizatoria del proyecto de la modernidad capitalista "mantenemos que la modernidad capitalista — que es como denominamos a este modelo específico de organización social-es incapaz, por sus propios medios, de responder a los graves retos globales actuales. La razón principal para mantener esta afirmación reside en que opinamos que la modernidad capitalista genera vulnerabilidad de manera estructural — siendo ésta, por tanto, parte de su propia esencia-, y como consecuencia de ello se ha convertido en un impedimento profundo para la seguridad y el bienestar de la humanidad. Con todo ello, nos sumamos al número creciente de autores y autoras que definen la crisis en clave civilizatoria (Acosta, 2010; Ceceña, 2010; Dierckxsens, 2008; Orozco, 2012; León I., 2010; Vega, 2009; Wallerstein, 2004; Valdés, 2009; Wilkinson, 2009; Fernández Durán, 2011), haciendo referencia a la necesidad de cuestionar el conjunto del proyecto modernizadon».

3 Tal y como afirma Fernández, "Hablamos de crisis civilizatoria, atravesamos una crisis multidimensional cuya génesis situamos en el propio modelo de sociedad global imperante, cuyos parámetros de civilización básicos desde hace más de dos siglos (progreso, individualismo, crecimiento económico capitalista y democracia liberal-representativa) y cuyos principios fuertes (ánimo de lucro, ganancia, acumulación), agudizados en la actual fase de globalización neoliberal, nos conducen a un callejón sin salida. Esto es fundamentalmente porque el propio sistema es incapaz de enfrentar esta situación en clave de bienestar, democracia, justicia y sostenibilidad, valores de segundo orden o incluso antagónicos con su propia naturaleza. Y es esta naturaleza la que lleva en su génesis una vulnerabilidad estructural que se manifiesta en un sistema de dominación múltiple que sufrimos las mayorias populares, de manera intensa pero desigual, $y$ en el que se articulan capitalismo, patriarcado, productivismo, colonialidad y democracia de baja intensidad. (...) Asistimos a una crisis que apunta a la raiz del sistema. Un sistema que de manera asfixiante y natural ha ido mercantilizando, dominando y arrebatando espacios a la democracia, a la humanidad, a la vida en definitiva, por lo que no puede ser reformado sino trascendido. Nos enfrentamos pues a un conflicto explícito entre la vida y el capital, en el que este último lamina las bases materiales de reproducción de la vida, se desarrolla sobre violencia y sobre crecientes desigualdades que afectan a grandes mayorias, y se muestra ingobernable a la hora de responder a criterios de justicia y sostenibilidadi. 
En este sentido, el movimiento campesino internacional organizado en $\mathrm{La}$ Vía Campesina (LVC) lleva décadas denunciando las grietas de este sistema (crisis alimentaria, crisis climática, crisis social) y proponiendo alternativas para su superación. Frente a quienes ante esta crisis están proponiendo una intensificación del desarrollismo, de la privatización y de la acumulación, La Vía Campesina y todas las organizaciones y personas que conforman lo que podemos nombrar como el movimiento por la soberanía alimentaria, ven la situación actual como de oportunidad y urgencia para hacer propuestas transformadoras, viables $\mathrm{y}$ reales en nuestros territorios.

Así, la soberanía alimentaria es una propuesta política que frente a la visión capitalista hegemónica de entender la alimentación como mercancía, defiende que eéta es un derecho, y confronta y propone alternativas al modelo agroindustrial. Este modelo, a partir de la nombrada como Revolución Verde ha pretendido convertir la agricultura y la alimentación en negocio, dentro de las lógicas de los mercados capitalistas, a través de la conformación de grandes cadenas de producción y distribución de alimentos, en manos de empresas transnacionales.

Así, podemos decir que el actual sistema agroalimentario capitalista, entendiendo por sistema agroalimentario el camino que va desde la producción de alimentos hasta el consumo de los mismos, está controlado por grandes empresas transnacionales, que utilizando múltiples estrategias en las diferentes fases de la cadena, llegan a definir qué y cómo se produce y también qué vamos a consumir ${ }^{4}$.

Para el modelo capitalista, y específicamente para este modelo agroindustrial, la naturaleza es otra mercancía más, una fuente inagotable de la que extraer recursos a los que poner valor monetario, un espacio para la explotación, el uso ilimitado, la privatización y la maximización de beneficios económicos. Así, la tierra, elemento central para la soberanía alimentaria, está en continuo peligro, porque se enfrenta tanto a las dinámicas promovidas por el modelo agroindustrial (concentración, intensificación, especulación...) como a los proyectos desarrollistas (urbanización, grandes infraestructuras, sistemas de transporte, extractivismo...) $)^{5}$.

\footnotetext{
${ }^{4}$ La variable del consumo resulta central, ya que se ha dado una progresiva globalización de las pautas de consumo, equiparando con la modernidad y el progreso, la disponibilidad permanente de estos alimentos disociados de los momentos de producción (determinadas frutas o verduras en cualquier momento del año), de cualquier lugar de procedencia (los llamados «alimentos kilométricos» o petroalimentos) y homogeneizando los gustos, sin valorar la diversidad cultural asociada a la alimentación.

5 Tal y como apunta el sindicato agroganadero EHNE-Bizkaia, «el aumento de las superficies de uso industrial, residencial, comercial e infraestructural ha tenido esencialmente dos consecuencias negativas para con la actividad agraria; por una parte, porque la está dejando sin tierra, elemento estructural para llevar a cabo una agricultura sustentable en términos físicos, medioambientales, sociales y económicos; y por otra, porque ha proporcionado que la tierra se vea sometida a la especulación urbanistica, pidiendo por ella unos precios desorbitados, y haciendo prácticamente inviable que aquellas personas que desean dedicarse a la actividad agrícola puedan acceder a la misma». Para más información, consultar página de EHNE-Bizkaia, Euskal Herriko Nekazarien Elkartasuna: http://www.ehnebizkaia.org/index.php/es
} 
El modelo agroindustrial, las cadenas agroalimentarias y las políticas desarrolladas en Europa, por ejemplo la Política Agraria Comunitaria (PAC) o las más recientes propuestas que se recogen en el Tratado Transatlántico de Comercio e Inversión entre Estados Unidos y la Unión Europea (TTIP, por sus siglas en inglés) ${ }^{6}$, han promovido la concentración de tierras en manos de corporaciones transnacionales para su uso intensivo y el monocultivo, el endeudamiento, la insostenibilidad y la desaparición de las pequeñas y medianas explotaciones y fincas ${ }^{7}$. Debemos añadir que en todo el mundo, muchas comunidades han sido expulsadas de sus tierras según los intereses de estas empresas, siendo violados sus derechos más fundamentales (Grain, 2009a).

Para el caso de las semillas, centrales también en la propuesta de la soberanía alimentaria, las empresas transnacionales han identificado el valor de las mismas para controlar la agricultura y la alimentación mundial. Así las semillas se han convertido en algo privado, en manos de pocas empresas, patentadas y, por tanto, se ha criminalizado su uso y producción libre. Asociada a esta desprotección de las semillas como bienes naturales y a todas las normativas y leyes que han permitido este lucrativo negocio, ha habido una pérdida de variedades y semillas tradicionales, siendo estas sustituidas por semillas comerciales priorizadas bajo criterios muy alejados de la biodiversidad y no adaptadas a los territorios. Las mismas empresas que comercializan las semillas son las que venden los fertilizantes y los agroquímicos para tratar plagas y enfermedades, productos que a su vez perjudican la salud de las personas productoras y consumidoras. Esta concentración y pérdida de diversidad se da también en las variedades y razas de animales (ETC Group, 2013) ${ }^{8}$.

Todo ello tiene consecuencias directas en promover una agricultura y ganadería menos resilientes y adaptadas a los entornos, más vulnerables, y en la pérdida de conocimiento y saberes tradicionales de los y las baserritarras, en conservación, reproducción, siembra e intercambio de semillas (La Vía Campesina, 2013). Para el movimiento campesino, perder la soberanía sobre las semillas, significa ceder definitivamente a las empresas transnacionales el poder de decidir qué podemos cultivar y qué podemos comer.

\footnotetext{
${ }^{6}$ Más información disponible en No al TTIP, http://noalttip.blogspot.com.es/p/documentos. html

7 Extraído del Documento Político de Etxalde Elikadura Burujabetza Munduan eta Euskal Herrian, disponible en: file://C:/Users/USUARIO/Downloads/Etxalde\%20elikadua\%20 burujabetza\%20munduan\%20eta\%20Euskl\%20Herrian.pdf

8 Según el último estudio realizado por ETC cuatro firmas en el mundo controlan el 58.2\% de las semillas, el $61.9 \%$ de agroquímicos, el $24.3 \%$ de fertilizantes, y el $53.4 \%$ de fármacos para animales. En la ganadería, cuatro empresas controlan el $97 \%$ de la investigación genética de aves de corral y dos tercios de la investigación y desarrollo en ganado porcino y reses.
} 
Por otro lado, y avanzando con este diagnóstico, podemos ver que la agricultura, tradicionalmente una gran productora de energía, se convierte con el modelo agroindustrial en una de las principales responsables del cambio climático: según LVC entre el 44 y el 57\% de las emisiones de gases de efecto invernadero $(\mathrm{GEI})$ están relacionadas con el sistema agroalimentario actual ${ }^{9}$. Este sistema requiere de mucha energía para mantenerse: más maquinaria; transporte de los alimentos y mercancías desde distintas partes del mundo; uso de agroquímicos y pesticidas, contaminantes también en su elaboración y derivados del petróleo; grandes extensiones de producción que requieren de un gran consumo de agua; pérdida de diversidad con la promoción de grandes zonas de monocultivos para la producción, por ejemplo, de biocombustibles; y dinámicas de acaparamiento de tierras en países del sur. Y, tal y como afirma LVC, son los pequeños campesinos/as, las comunidades rurales, los y las baserritarras, quienes más directamente sufren el cambio climático $^{10}$.

Podemos por tanto definir este modelo agroindustrial como altamente dependiente: por el modelo de producción, intensivo, especializado, tecnificado, que convierte a los y las baserritarras en un sector que necesita de esos productos provenientes de las grandes empresas (semillas, abonos, fertilizantes, maquinaria...), escapando a su control el precio y el funcionamiento de los mismos; por el uso intensivo de fuentes de energía y consumo de bienes naturales; por la especialización y homogeneización de las producciones, acabando con la diversidad de los territorios y aumentando su dependencia externa. Y, finalmente, dependiente también por la orientación que se le da a las producciones hacia el mercado mundial, "haciendo que los agricultores y agricultoras pierdan el control sobre los precios, que quedan en manos de las distribuidoras. Pero también generan un modelo centralizado de distribución, en el que los alimentos recorren grandes distancias por todo el planeta desde la producción hacia el lugar donde se procesan o envasan, y de ahi hasta el lugar de consumo final. Este modelo agroalimentario requiere de grandes infraestructuras de transporte y logistica, y de la destrucción de importantes cantidades de alimentos que no encuentran mercado, o no a los precios requeridos por las cadenas de distribución» (López y Llorente, 2010).

Además, y continuando con este análisis del modelo agroindustrial desde una mirada integral, no podemos obviar que como parte del sistema capitalista necesita del patriarcado para su funcionamiento, está por tanto construido sobre las relaciones de poder de los hombres sobre las mujeres. Así podemos ver

9 Extraído del Documento Político de Etxalde Elikadura Burujabetza Munduan eta Euskal Herrian, disponible en: file://C:/Users/USUARIO/Downloads/Etxalde\%20elikadua\%20 burujabetza\%20munduan\%20eta\%20Euskl\%20Herrian.pdf

10 Para más información consultar el documento, "La agricultura campesina es una solución verdadera a la crisis climática", Llamado a la acción de LVC para la Cop21, disponible en: http://viacampesina.org/es/index.php/acciones-y-eventos-mainmenu-26/cambios-climcos-y-agrocombustibles-mainmenu-79/2468-la-via-vampesina-llamado-a-la-accion-para-la-cop21-en-paris 
que las mujeres campesinas, tal y como apuntan múltiples estudios, han sufrido y sufren la invisibilización y el no reconocimiento de su trabajo y experiencia (Caro, 2013), y este desconocimiento de su situación específica, necesidades e intereses ha marcado la definición de muchas de las propuestas realizadas para el mundo campesino: desigual acceso a la tierra, a los derechos laborales, a las ayudas y a la financiación, sobrecarga de trabajo por la ausencia de corresponsabilidad en las tareas de cuidado, menor participación socio-política, etc., son solo algunos de los rostros que muestra esta desigualdad. Cuestiones que se agudizan con este modelo de producción agroindustrial, que según muchas autoras, está definido por y para un modelo de masculinidad hegemónica, ya que «se trata de una agricultura basada en la economía de mercado, financiera y monetaria, que se desarrolla por los hombres en el ámbito público, único valorado y reconocido, y que se rige por la lógica de acumulación (...), siendo así, y sin ánimo de esencializar, fundamentalmente las mujeres las encargadas de mantener y defender un modelo enfrentado al agroindustrial (García 2012; Arriola et al., 2009) ${ }^{11}$.

Para finalizar con este breve diagnóstico, hacemos referencia a tres cuestiones que permiten comprender este modelo agroindustrial, y que son señas de identidad de la modernidad capitalista: el individualismo, la democracia de baja intensidad y la privatización de los conocimientos. Este sistema se basa en una visión individualista de las personas, siempre centradas en la búsqueda de su satisfacción, y encontrando en el mercado y en el consumo el ámbito prioritario para ello. En segundo lugar, la política se ve como algo que sucede en las instituciones en las cuales se delega toda la responsabilidad y el poder en cada período electoral, y las personas cada vez tienen una menor participación en la construcción y la organización de eso colectivo. En tercer lugar, la información y los conocimientos también se van privatizando, tecnificando y concentrando en pocas manos, no garantizando el acceso libre y el poder sobre los mismos por parte de todas las personas.

Desde el mundo campesino siempre se ha denunciado cómo el modelo agroindustrial se ha basado en el no reconocimiento de los saberes y prácticas tradicionales campesinas y por tanto, en su abandono, considerándolos algo

${ }^{11}$ El modelo de producción agroindustrial «es una agricultura que se sustenta en la economía doméstica, de los cuidados y de la afectividad, desarrolladas por las mujeres en el ámbito privado, invisibilizadas y no valoradas. Fueron los hombres quienes, en gran medida, transformaron su actividad agraria campesina por una cuyo objetivo era ganar dinero; mientras que muchas mujeres siguieron desarrollando actividades campesinas, gracias al trabajo relacionado con el sustento de la familia y de los cuidados, manteniendo su función social, ambiental, cultural y productiva en el medio rural, (García, 2012). Otras autoras sostienen que las mujeres campesinas, "están por lo general más ligadas a la tierra que los varones y ponen en práctica una agricultura más variada y diversificada en contraposición a la uniformidad y homogeneidad que impera en los procesos de agricultura industrial e intensificada» (Arriola et al., 2009). 
atrasado (Grain, 2009b) ${ }^{12}$; en la tecnificación de las prácticas, alejando los procesos de investigación de la tierra y de las personas conocedoras de la misma; en la aplicación de criterios como la maximización de beneficios y el máximo rendimiento a la producción de alimentos y en la construcción de relaciones sociales y de intercambio acordes con esto; y en el debilitamiento de formas de aprendizaje características de las poblaciones rurales y campesinas. Se trata por tanto de un modelo en el cual, las empresas transnacionales como actores fundamentales del sistema capitalista, no solo detentan el poder económico y político, sino también el cultural, ya que «juegan un papel fundamental en la reproducción simbólica del sistema, convirtiéndose en sujetos activos en defensa de una civilización individualista, consumista, fragmentada y despolitizada. De esta manera, han entendido con claridad que su legitimación depende de los imaginarios colectivos, de los valores imperantes, para lo cual han llevado la cultura a su terreno (mercantilizándola en la medida de lo posible), a la vez que han diseñado, impulsado y generalizado un formato universal de sociedad, de ciudadanía global, y de saber y conocimiento, adaptado a la primacía del crecimiento capitalista y a la democracia de baja intensidad» (Fernández, 2015).

Por tanto, y para finalizar con este primer apartado, podemos concluir que nos encontramos ante un modelo, el agroindustrial, que no garantiza el acceso a la alimentación y que produce vulnerabilidad ecológica, climática, alimentaria, social y económica. Frente a esto, veamos en el siguiente punto, cuáles son las propuestas de la agenda de la soberanía alimentaria.

\subsection{Las principales propuestas de la soberanía alimentaria como enfoque integral, abierto y alternativo}

Si las anteriores líneas nos han permitido exponer algunos de los elementos que caracterizan la producción de alimentos y la agricultura en el actual modelo capitalista, ver cómo la privatización atraviesa todo este ámbito y los múltiples impactos que este modelo tiene para las personas y los territorios, en este segundo apartado queremos presentar los principales elementos que articulan la agenda de la soberanía alimentaria, ideas que entendemos nos permitirán argumentar por qué defendemos que se trata de una propuesta imprescindible que antepone la vida y su reproducción, frente a la lógica biocida (Herrero, 2015) del sistema capitalista.

12 "Hace algo menos de cien años se dijo -y se nos sigue diciendo-que ser campesino o indígena es sinónimo de ignorancia, superstición, atraso. Desde los centros de investigación, desde las universidades y especialmente desde las escuelas nos hacen la propaganda de que los únicos que saben son los investigadores, los agrónomos, los profesores. Miles de años de observación cuidadosa, relaciones de cuidado y afecto, búsqueda colectiva y aprendizaje mutuo tenían que olvidarse para dar cabida a lo aprendido en los campos de experimentación bajo condiciones controladas. Se inventaron los conceptos de "extensión" y "transferencia», para dejar claro que el conocimiento se producia en determinados lugares — muy reducidos_ y el resto del planeta debia recibirlo pasivamente». 
Definimos la soberanía alimentaria como un enfoque político que se basa en el derecho de los pueblos a establecer su propio sistema alimentario, y frente a un modelo único basado en la centralidad de los mercados, la privatización de la vida y la generación de desigualdades, este enfoque propone colocar la vida y la alimentación en el centro y construir colectivamente otros modelos sociales y económicos que respondan a esta apuesta.

La Vía Campesina dice en su Declaración de Nyèlèni $(2007)^{13}$, que entiende por soberanía alimentaria lo siguiente:

La soberanía alimentaria es el derecho de los pueblos a alimentos nutritivos y culturalmente adecuados, accesibles y producidos de forma sostenible y ecológica, y su derecho a decidir su propio sistema alimentario y productivo. Esto pone a aquellos que producen, distribuyen y consumen alimentos en el corazón de los sistemas y políticas alimentarias, por encima de las exigencias de mercados y empresas. Defiende los intereses de, e incluye a, las futuras generaciones. Nos ofrece una estrategia para resistir y desmantelar el comercio libre y corporativo y el régimen alimentario actual, y para encauzar los sistemas alimentarios, agrícolas, pastoriles y de pesca para que pasen a estar gestionados por los productores y productoras locales. La soberanía alimentaria da prioridad a las economías locales y a los mercados locales y nacionales, y otorga el poder a los campesinos y a la agricultura familiar; la pesca artesanal y el pastoreo tradicional, y coloca la producción alimentaria, la distribución y el consumo sobre la base de la sostenibilidad ambiental, social y económica. La soberanía alimentaria promueve el comercio transparente, que garantiza ingresos dignos para todos los pueblos, y los derechos de los consumidores/as para controlar su propia alimentación y nutrición. Garantiza que los derechos de acceso y a la gestión de nuestra tierra, de nuestros territorios, nuestras aguas, nuestras semillas, nuestro ganado y la biodiversidad, estén en manos de aquellos que producimos los alimentos. La soberanía alimentaria supone nuevas relaciones sociales libres de opresión y desigualdades entre los hombres y mujeres, pueblos, grupos raciales, clases sociales y generaciones.

De esta definición, amplia, rica y en permanente construcción extraemos tres ideas que, en nuestra opinión, son fundamentales: la soberanía; la construcción popular de las alternativas; y la centralidad de las economías campesinas y la agroecología. Exponemos estas claves brevemente a continuación:

13 Declaración fruto del Foro celebrado en Bamako, Mali en febrero de 2007, y nombrado como Foro de Nyéléni, en el que más de 700 personas de todo el mundo, de diferentes organizaciones de productores/as, campesinas, pueblos indígenas y movimientos sociales, se reunieron para fortalecer la comprensión y allanar el camino hacia una implantación activa de la soberanía alimentaria. Este Foro cumplió con su objetivo de reunir a pueblos de todo el mundo, representando un amplio abanico de sectores que luchan por la soberanía alimentaria. Y se debatieron los temas relacionados con la soberanía alimentaria buscando: profundizar el conocimiento colectivo; fortalecer el diálogo entre y a través de los sectores y los grupos de interés; y elaborar estrategias comunes y una agenda de acciones conjuntas. Más información sobre este Foro y el Informe completo del mismo disponibles en: http://www.nyeleni.org/spip.php?article341 
- Clave 1, centralidad del concepto de soberanía: La soberanía alimentaria fundamenta toda su propuesta en el derecho a decidir, en la capacidad de los pueblos para definir su sistema alimentario y para desarrollar las políticas que consideren para establecer qué y cómo se produce, qué y cómo se distribuye, qué y cómo se consume. Frente a la centralidad otorgada a los mercados, a las grandes empresas, a los organismos internacionales o a los estados y sus intereses geopolíticos, la soberanía alimentaria reivindica la autonomía de los pueblos y personas para construir alternativas sociales, económicas y políticas, adecuadas a sus contextos y realidades y que tengan por objetivo final no la maximización de los beneficios sino el bienestar en sentido amplio.

- Clave 2, importancia de la construcción popular de las alternativas: La soberanía alimentaria es un enfoque que proviene del movimiento campesino internacional. No nace ni desde el ámbito académico ni desde los organismos internacionales o administraciones. Nace y se construye desde el propio campesinado, se construye desde la experiencia. Esto supone, que es una propuesta política para la acción y la práctica; que se basa en la promoción de procesos de empoderamiento y generación de conocimiento critico; y que apuesta por la construcción colectiva y popular de las alternativas.

- Clave 3, economías campesinas y agroecología como pilares de la propuesta: la soberanía alimentaria basa esta construcción de modelos alternativos en la defensa, en primer lugar, de las economías campesinas y en los sistemas de producción, distribución y consumo que estén vinculadas a estas. Y, en segundo lugar, en la agroecología como enfoque que debe definir esta construcción de alternativas, tanto desde su dimensión más técnica como en los ámbitos socioeconómico y sociopolítico (Altieri, 2000; Sevilla, 2006) ${ }^{14}$.

No podemos detenernos en este artículo en exponer con detenimiento los dos últimos conceptos vinculados a las claves de la soberanía alimentaria (economías campesinas y agroecología), pero sí apuntar que son dos propuestas ínti-

14 La agroecología sigue siendo un concepto muy rico y en construcción, que va más allá de las prácticas de producción agroecológicas. La agroecología «ve el proceso agrícola como un sistema integrado, por aspectos ambientales, económicos, sociales y culturales; y su finalidad no es sólo incrementar la productividad de uno de los componentes; sino, de optimizar el sistema como un todo y mantener la sustentabilidad en el tiempo y espacio" (Altieri, et al, 2000). La agroecología puede definirse como "el manejo ecológico de los recursos naturales a través de formas de acción social colectiva que presentan alternativas al actual modelo de manejo industrial de los recursos naturales mediante propuestas surgidas de su potencial endógeno, que pretenden un desarrollo alternativo desde los ámbitos de la producción y la circulación alternativa de sus productos, intentando establecer formas de producción y consumo que contribuyan a encarar la crisis ecológica y social, y con ello a enfrentarse al neoliberalismo y a la globalización económica» (Sevilla, 2006). 
mamente unidas. La agroecología, de manera muy general, es un enfoque multidisciplinar que nos remite a una triple dimensión de sostenibilidad: ecológica (conocimientos tradicionales, sistemas integrados y diversos, producción extensiva, técnicas ecológicas, manejo sustentable de bienes naturales, eficiencia y bajo consumo energético, etc.); socio-económica (la puesta en marcha de los llamados «sistemas locales agroalimentarios», promoviendo sistemas de distribución justos, locales, vinculando productores/as y consumidores/as, etc...); y una dimensión socio-política (que pone el acento en la necesidad de transformar las relaciones de poder en las que se da esa producción y enfrentar las distintas desigualdades, de género, edad, clase, norte-sur, promoviendo una construcción colectiva de estas alternativas).

Si entendemos las economías campesinas como sistemas de producción de menor tamaño, locales, diversos, basados en sistemas de distribución y comercialización de cercanía, y donde priman valores como la cooperación y la autogestión, podemos afirmar que son las que mayor capacidad y potencialidad muestran para desarrollar todas las dimensiones del modelo agroecológico $\left(\right.$ Gallar, 2013) ${ }^{15}$. Por tanto, se trataría de dos elementos que deben ser entendidos de manera conjunta, y centrales para la propuesta de la soberanía alimentaria.

Consideramos que estas tres claves explican de manera muy resumida los elementos centrales de la agenda de la soberanía alimentaria. Además, e intentando dar respuesta a por qué defendemos que se trata de una propuesta contrahegemónica de primer orden y que enfrenta, con propuestas reales que vinculan reflexión teórica y práctica, el poder de las empresas transnacionales y al modelo alimentario agroindustrial, proponemos entenderla como un enfoque integral, abierto y alternativo.

Integral, porque partiendo de la defensa de la alimentación no como mercancía sino como derecho fundamental, es un enfoque que aborda de manera crítica las relaciones y dinámicas que se dan en el resto de ámbitos sociales y económicos. Así podemos decir que no se trata solo de transformar lo que comemos, sino sobre todo de transformar la realidad en la cual está inserta esta producción y ese consumo de alimentos. Tal y como hemos expuesto anteriormente, se proponen transformaciones en múltiples esferas y niveles, desde los valores y principios en lo que se articulan nuestras sociedades, la manera de construir los modos de organización, lo público y las políticas y normativas concretas, hasta la propuesta de alternativas en lo más micro.

15 "Podemos caracterizar la economía campesina como un modelo que tiene como bases el atributo de la autonomía en las dimensiones ecológica, económica y cultural; que tiende a la equidad en el acceso y aprovechamiento de los recursos locales; que posee altos niveles de producción, suficientes para la satisfacción de necesidades básicas de sus miembros; es una economía no monetaria y no orientada al beneficio, sino a la reproducción; y es una economía no depredadora y no explotadora de personas». 
Es una agenda también abierta, porque está en permanente construcción y porque dialoga y se nutre de otras propuestas políticas, como la economía feminista, el ecofeminismo, la economía social y solidaria, la economía ecológica, el decrecimiento, la economía del bien común o el Buen Vivir, entre otras. A nuestro entender, este es un elemento destacable de la agenda de la soberanía alimentaria, un enfoque sólido, pero no cerrado ni excluyente. Una propuesta que ha ido mostrando, en los últimos ańos, la capacidad de contagiarse, tanto en sus contenidos más teóricos como en sus prácticas, de esas otras agendas alternativas que mencionábamos ${ }^{16}$.

Y por último, se trata de una propuesta alternativa, porque frente a ese modelo hegemónico, que decíamos genera pobreza, exclusión, superación de los límites del planeta, desigualdad, etc., este enfoque propone un modelo social y económico alternativo basado en la sostenibilidad ecológica, económica y social. La soberanía alimentaria plantea descentrar los mercados y la acumulación y construir otros modelos en los cuales la sostenibilidad de la vida en todas sus dimensiones sea el objetivo a perseguir. Además, frente a la idea de que no hay otras maneras posibles de hacer, el enfoque de la soberanía alimentaria defiende que sí es posible construir alternativas emancipadoras.

Tomando todas estas ideas como paraguas, avanzamos en la segunda parte de este artículo algunas ideas para abordar las políticas públicas como una herramienta que puede favorecer el desarrollo de esta agenda en el ámbito local, y por tanto, facilitar la transición hacia esas alternativas de vida.

\section{La construcción de otros modelos de vida en los territorios: políticas públicas locales como herramienta para la transformación ${ }^{17}$}

Pasamos en esta segunda parte del artículo a exponer por qué consideramos que los territorios y lo local son ámbitos fundamentales para la puesta en mar-

16 En la práctica esto se puede concretar, por ejemplo, en cómo la soberanía alimentaria, o muchas de sus propuestas, están siendo incorporadas a las agendas y reivindicaciones de diferentes movimientos u organizaciones sociales, o en los diferentes espacios de diálogo y alianza que el movimiento campesino lleva años tejiendo con organizaciones feministas, indígenas, urbanas, decrecentistas... Por incorporar dos experiencias recientes que han tenido lugar en Euskal Herria, mencionamos la Acción Internacional de la Marcha Mundial de las Mujeres 2015, que definió como uno de sus ejes la soberanía alimentaria, el intercambio de semillas (más información en http://www. pikaramagazine.com/2015/09/en-marcha-hasta-que-todas-seamos-libres/) y la presencia y participación que la soberanía alimentaria y el movimiento campesino han tenido en el proceso de Alternatiben Herria, http://alternatibenherria.eus/?lang=es

17 Apuntar que para La Vía Campesina, tal y como se recoge en muchos de sus documentos, la construcción de políticas públicas que puedan responder a la agenda de la soberanía alimentaria siempre ha sido un campo de lucha y uno de los elementos importantes de su agenda. 
cha de la agenda de la soberanía alimentaria, y desde qué enfoque creemos se deben abordar las políticas públicas, como herramienta que puede facilitar esta transformación.

\subsection{Intersección de soberanías: propuesta en construcción para mirar nuestros territorios}

Tal y como venimos apuntando en este artículo, la soberanía alimentaria propone una transformación integral en el modo de organización social, una transformación necesariamente ligada a los territorios y a las vidas de las personas. Para la soberanía alimentaria por tanto es central la tierra, el territorio, lo local, como ámbito estratégico para la construcción colectiva y la puesta en marcha de alternativas de vida.

El territorio es también un ámbito en disputa, en el cual podemos encontrar diversidad de propuestas y actores, diversidad de intereses y visiones. Debemos aclarar que entendemos lo local no desde una perspectiva localista ni reduccionista, sino siempre en necesaria interacción y relación con lo global. No podemos analizar nuestros contextos y realidades más cercanas, sin entender cómo estas están afectadas por causas globales estructurales. Nuestras propuestas deben partir, tanto en el análisis del modelo hegemónico generador de múltiples desigualdades, como en las alternativas al mismo existentes, de esta idea de interdependencia. La Vía Campesina es, en sí misma, un ejemplo perfecto para entender esta propuesta de articulación local-global.

Así podemos decir que el ámbito local, el territorio, a pesar de estar condicionados por normativas, regulaciones y actores que operan en otros niveles de decisión, son un ámbito que permite desarrollar una mayor autonomía por parte de las comunidades. Además, son ámbitos estratégicos para relocalizar y desarrollar esos otros sistemas agroalimentarios sustentables ecológica, social, política y económicamente, ligados a la tierra, a las formas de vida campesinas, a la cercanía, y en los cuales resulta central el vínculo y la corresponsabilidad entre quienes producen y quienes consumen. Permiten por otro lado el aterrizaje de este enfoque político en proyectos concretos, que surgen desde y para los contextos y realidades en los que se formulan, y la construcción colectiva de estos. Por último, los territorios y lo local son espacios donde más fácilmente podemos entender que el bienestar o felicidad de las personas no está directamente vinculado con indicadores de crecimiento económico macro, sino que tienen que ver con ese vivir bien en sentido amplio, con las capacidades, la libertad, la autonomía, el tiempo, etc., elementos que se pueden incorporar a los proyectos, en los cuales lo relacional y la comunidad adquieren un papel relevante.

Desde estas premisas, hemos tratado de responder a las siguientes cuestiones: si quisiéramos construir una propuesta colectiva alternativa desde lo local, desde la soberanía alimentaria, y que intentara desarrollar todas las claves ante- 
riormente identificadas, ¿Qué ámbitos incluiría? ¿Qué ámbitos de soberanía tenemos que conquistar para poder ir concretando y construyendo ese otro modelo de sociedad, de vivir bien, que responda a estas claves y principios? ¿Sobre qué ejes podemos articular este proyecto alternativo, respondiendo a esa necesaria mirada integral?

Intentando dar respuesta a estas cuestiones surge la propuesta del enfoque de intersección de soberanias, como un posible camino para abordar la definición de nuestros proyectos colectivos locales de vida. Frente a la tendencia a mirar la realidad, las desigualdades y las agendas y propuestas de manera sectorial, hemos tratado de presentar un enfoque que responda a la necesaria visión holística de la soberanía alimentaria y permitirnos avanzar en la integralidad de las propuestas.

Este enfoque plantea que nuestros proyectos de buen vivir locales, construidos desde la propuesta política y enfoque de la soberanía alimentaria, nos remiten a tener derecho a decidir y proponer alternativas en, al menos, los siguientes cinco ámbitos: bienes naturales; energía; sistemas alimentarios locales; autonomía y derechos de las mujeres; y organización de lo común y conocimiento libre.

Antes de presentar algunos elementos de cada ámbito de soberanía identificado, nos gustaría destacar dos ideas. En primer lugar, lo central del concepto de soberanía. En los cinco ámbitos identificados consideramos fundamental reivindicar el derecho a decidir y construir alternativas de las personas y pueblos, y encontramos además grandes potencialidades y retos para el avance de la propuesta de la soberanía alimentaria. Así hemos preferido nombrarlos como soberanías, como ámbito de conquista, como ámbitos en los cuales, desde la autonomía y empoderamiento, "detraer espacios y recursos al capital» y proponer modelos alternativos al hegemónico "bajo otras lógicas de solidaridad y reciprocidad» (P. Orozco, 2012) ${ }^{18}$.

En segundo lugar, destacar que este enfoque de intersección de soberanías está en construcción, es una propuesta que debe ser alimentada y profundizada,

18 Tal y como apuntan diversas autoras feministas, como Amaia Pérez Orozco, "(...) De qué estructuras socioeconómicas nos dotamos para articular una responsabilidad colectiva en la reproducción de las condiciones de posibilidad para esa vida que merece la pena ser vivida. De nuevo, el sistema actual no nos sirve, por esa contradicción estructural entre el proceso de valorización de capital y el proceso de sostenibilidad de la vida (...) como lo afirma Antonella Picchio, el capitalismo es una "economía de muerte" $o$, en palabras de Herrero (2010b), es un sistema "biocida». La responsabilidad de sostenerla está privatizada, feminizada e invisibilizada. De aqui se abren múltiples debates, y, al menos, dos certezas: la propuesta no es dejar esa responsabilidad en los mercados capitalistas; estos no pueden ser la estructura socioeconómica priorizada, sino que, antes al contrario, han de tender a desaparecer. A la par, esa responsabilidad ha de ir democratizándose, colectivizándose y des-feminizándose. Si bien pueden parecer afirmaciones excesivamente amplias y abstractas, de ellas se deriva un primer movimiento estratégico fundamental: detraer recursos de la lógica del capital, para poder ponerlos a funcionar bajo otras lógicas económicas (de reciprocidad y solidaridad) en estructuras económicas democráticas». 
pero que consideramos puede darnos una posible mirada amplia, integral, que nos permita transitar hacia la definición de esos otros modelos alternativos.

Por tanto, tomando en consideración los tres pilares de la propuesta de la soberanía alimentaria (derecho a decidir, construcción popular y agroecología y economías campesinas), y entendiendo que se trata de una propuesta integral, abierta y alternativa, exponemos a continuación algunas de las ideas y objetivos propuestos para cada ámbito de soberanía.

\subsubsection{Soberanía sobre los bienes naturales}

Tierra, bosques, agua, semillas, etc., resultan fundamentales para la soberanía alimentaria. Frente a la actual privatización y especulación con los mismos, la soberanía alimentaria defiende que son fuente de diversidad y sostenibilidad, y por tanto, hay que cuidarlos y protegerlos. Dejar de verlos como meras mercancías para entenderlos como bienes. Debemos para ello promover una visión de nuestros territorios no solo desde su perspectiva productiva o económica, sino también ecológica, cultural y política, y abordar desde esa mirada la ordenación de los mismos. Consideramos que hay que promover procesos colectivos y participativos, en los que se definan desde la comprensión de los territorios como espacio natural, ecológico, económico y político, qué necesidades tenemos, cómo podemos y queremos satisfacerlas y cómo podemos hacer esto en clave de sostenibilidad, equidad y justicia. Las economías campesinas y la agroecología proponen unas dimensiones centrales para esa ordenación.

Así, de todos los objetivos posibles, priorizamos cuatro que nos pueden permitir avanzar en términos de soberanía en este ámbito:

- Defender el carácter comunal o público de estos bienes, y protegerlos frente a los procesos de especulación, privatización y abandono.

- Ordenar el control, uso y gestión de los bienes naturales en clave de sostenibilidad ecológica, económica y política y desde procesos participativos.

- Para el caso de la tierra, recuperar, defender y redistribuir la tierra y promover la producción desde el enfoque de la soberanía alimentaria.

- Promover la diversidad agrícola y ganadera, y la defensa de variedades y razas propias y no modificadas.

\subsubsection{Soberanía energética}

Frente a la actual crisis climática y energética, la soberanía alimentaria es una alternativa real. No se trata de tomar medidas puntuales dirigidas a producir más energía desde fuentes alternativas para seguir aumentando el consumo, sino que estamos en un momento en el cual la transformación del modelo, 
desde la apuesta por la soberanía y la construcción participada, es ya una necesidad. Transformar el modelo de consumo, reorganizar la energía con criterios de eficiencia, promover la autonomía energética y las energías de fuentes alternativas y renovables, reducir residuos, reutilizar, reciclar, y avanzar hacia un modelo público de producción, gestión y distribución de energía pueden ser alguno de los caminos. La relocalización de la producción y el consumo de alimentos y la producción agroecológica pueden sin ninguna duda ayudar a reducir el consumo energético.

En este caso proponemos avanzar en los siguientes objetivos:

- Transformar y reducir el actual modelo de consumismo energético.

- Promover la autonomía energética de los municipios y comarcas.

- Promover el empleo de energías alternativas.

- Avanzar hacia un modelo público de energía: producción, gestión, distribución.

- Reducir la generación de residuos y gestionar los mismos de manera más sostenible.

\subsubsection{Soberanía sobre los sistemas alimentarios locales}

En este ámbito de soberanía hacemos referencia a lo que tiene ver con la producción, transformación, distribución, intercambio, comercialización y consumo de alimentos. Tomando el principio de la alimentación como derecho, la construcción de estos sistemas alimentarios locales se basa en dos conceptos fundamentales: la (re)campesinización y la (re)localización, o siguiendo nuestro marco teórico, la apuesta por la agroecología y las economías campesinas, como pilares fundamentales de estos sistemas alternativos (VSF, 2013) ${ }^{19}$. Por lo tanto, podemos decir que promueven la autonomía y soberanía para decidir dónde y cómo se producen, transforman y comercializan los alimentos, priorizando los territorios, lo local, la producción ecológica, las relaciones de cooperación y los canales cortos de comercialización; y entre quiénes definimos estos sistemas alternativos, con la participación activa de diferentes personas y colectivos, (productoras, consumidoras, colectivos y organizaciones sociales, administración pública), entendiendo el consumo como una herramienta de transformación, compartiendo responsabilidades y construyendo relaciones de cercanía entre productores/as y consumidores/as, entre el ámbito rural y el urbano.

19 Esta sería la base para construir esos otros sistemas alimentarios locales, caracterizados según Di Masso, por "transitar de un sistema agroalimentario convencional (globalizado) hacia un sistema agroalimentario (re)localizado. Esta relocalización conllevaria una reconexión (entre producción y consumo), una redefinición (de valores) y una redistribución (del valor añadido) como objetivos básicos. Este término por tanto captura una dinámica de re-arraigo de los alimentos en su contexto natural y social que abriga y fomenta relaciones de proximidad y conectividad». 
Para este ámbito de soberanía proponemos los siguientes objetivos:

- Avanzar hacia una producción de alimentos local, diversificada, descentralizada y agroecológica en los municipios y comarcas.

- Promover un modelo de transformación local o comarcal de estos alimentos.

- Construir de manera colectiva, circuitos cortos de comercialización de alimentos, centrados en la compra local, de cercanía, agroecológica y de temporada, implicando tanto a las personas individuales como a la administración pública (en su papel de promotora y de consumidora).

\subsubsection{Soberanía y autonomía de las mujeres ${ }^{20}$}

Tal y como apuntábamos en páginas anteriores, consideramos que resulta necesario impulsar diversas estrategias para promover la soberanía y autonomía de las mujeres como elemento central de una propuesta alternativa como la soberanía alimentaria. Desde la visibilización y el reconocimiento de la realidad, de la situación y posición de las mujeres agricultoras y rurales, desde su diversidad, y el establecimiento de las medidas necesarias para mejorar ésta; dar valor a sus conocimientos y saberes para la construcción de la soberanía alimentaria y su papel fundamental para otro modelo de agricultura sostenible; promover procesos de empoderamiento de las mujeres campesinas, entendidos como la toma de conciencia individual y colectiva de su situación de desigualdad, de las causas que la generan, y de su organización para superarlas; hasta potenciar el trabajo en redes y alianzas de mujeres, siendo necesario establecer vínculos entre mujeres urbanas y rurales para luchar por objetivos comunes.

Así, en este ámbito de soberanía, proponemos abordar los siguientes objetivos:

- Visibilizar y reconocer la situación y posición de las mujeres baserritarras, desde la diversidad y en diferentes ámbitos como el acceso y control sobre recursos y bienes, participación socio-política, vidas libres de violencia, cuidados como derecho, etc. y avanzar en las medidas necesarias para mejorar éstas.

- Valorar y reconocer sus conocimientos y aportes, fundamentales para la construcción de modelos de producción de alimentos sostenibles.

- Promover procesos de empoderamiento y la organización de las mujeres baserritarras.

${ }^{20}$ Hemos decidido definir un ámbito específico de soberanía y autonomía de las mujeres, porque consideramos que la confrontación con el patriarcado es una cuestión fundamental para la soberanía alimentaria y para construir propuestas sociales, económicas y políticas alternativas a la actual y que la situación de desigualdad de las mujeres debe, todavía a día de hoy, ser reconocida, denunciada y enfrentada. Esta decisión no excluye tomar en consideración la incorporación de la perspectiva de género y el análisis feminista en el resto de ámbitos de soberanía abordados. 
- Avanzar en la generación de redes y alianzas entre las mujeres urbanas y rurales.

\subsubsection{Soberanía sobre la organización de lo común y conocimiento libre}

En este último ámbito de soberanía hacemos referencia a cuestiones relacionadas con la organización de lo común, la construcción de alternativas y el lugar central que ocupan el conocimiento y la información en todo esto. Así, se trata como decíamos anteriormente, de disputar el poder cultural a las empresas transnacionales, de combatir esa idea de un único modelo posible, del sentido común individualista y de la privatización tanto de la información, de los conocimientos como de la política o la organización de lo colectivo. Entendemos que no será posible construir esos otros proyectos de vida alternativos sino se aborda desde la generación de conciencia crítica de las personas y pueblos, y la comprensión de la centralidad de apropiarnos de la organización de lo colectivo. Por tanto, conocimientos libres y diversos y el acceso a la información resultan fundamentales. Resulta central ampliar y redefinir los marcos en los cuales se han insertado los procesos de toma de decisiones, priorizando la construcción y el debate colectivo sobre las posibles alternativas; y promoviendo espacios y relaciones basadas en la solidaridad y el intercambio.

Así podríamos definir en este último ámbito de soberanía tres grandes objetivos:

- Avanzar hacia la comprensión de los conocimientos y la información como bien común.

- Promover espacios para el debate, la reflexión y la construcción colectiva de las alternativas.

— Favorecer la construcción de relaciones de cooperación, colaboración y solidaridad en los territorios.

Esta sería por tanto, y de manera resumida, la mirada que proponemos lanzar a nuestros territorios. Avanzamos en el siguiente punto el papel que las políticas públicas pueden tener para el desarrollo de este marco.

\subsection{Las políticas públicas como herramienta: algunas claves para desarrollar este enfoque alternativo}

Comenzar diciendo que entendemos las políticas públicas locales como un ámbito especialmente interesante para el desarrollo de la agenda de la soberanía alimentaria. Una herramienta, posiblemente limitada, pero en la que podemos encontrar actualmente iniciativas y procesos con gran potencialidad, laboratorios de experiencias de las que se pueden extraer muchos aprendiza- 
jes $^{21}$. En nuestra opinión, y asumiendo la necesidad de diversas estrategias y líneas de trabajo para el desarrollo de la agenda de la soberanía alimentaria, las políticas públicas locales pueden ser un elemento tractor fundamental para ir avanzando en la transición hacia esos otros modelos deseados.

Así nuestra mirada a las políticas públicas es desde una apuesta por otra manera de entender lo público, más cercana al bien común, y alejada de la creciente privatización de las vidas, de los derechos, de los bienes naturales, etc. Lo público, en definitiva, como un espacio del que apropiarse colectivamente. Partimos desde luego de una mirada crítica al actual modelo desde el que se construyen, se piensan, diseñan y desarrollan esas políticas públicas. Asumir la soberanía alimentaria como enfoque que oriente la construcción de otras propuestas políticas, al igual que otros enfoques de carácter transformador, supone una revisión de cómo se ha entendido hasta ahora la participación, los procesos de toma de decisiones, la gestión y la organización de lo público. Ampliar y redefinir los marcos a los cuales se ha limitado la capacidad de decisión de las personas y pueblos. Supone entender que frente a una visión tecnocrática y supuestamente neutra de los poderes públicos, estos pueden ser aliados para el desarrollo de agendas alternativas y transformadoras, o grandes obstáculos para la misma.

Este planteamiento implica grandes retos y desafíos, y en lo concreto, supone asumir que no podremos hablar de transformación real si la administración va sola; que podemos realizar muy buenas propuestas técnicas desde nuestros municipios que fracasen por no haber contado con la participación y visión de las personas directamente implicadas; que la administración puede tener un papel importante para facilitar y promover los cambios; que deberemos tratar de dar respuesta a las urgencias, a las necesidades reales e inmediatas, y ser capaces de valorar cómo esas respuestas nos alejan o acercan de ese horizonte de transformación; que hay experiencias autónomas, que seguirán siendo eso, autónomas, pero de las cuales tenemos mucho que aprender; que existen riesgos evidentes de burocratización de los procesos a los que tendremos que hacer frente; y que aterrizar un enfoque como la soberanía alimentaria, en propuestas concretas, requiere aunar esfuerzos y entender que es una tarea en permanente construcción.

Presentamos a continuación algunos principios que consideramos necesarios para abordar la puesta en marcha de esas otras políticas públicas desde lo local.

1. Asumir compromisos políticos claros que van más allá de lo meramente discursivo. Supone asumir el reto de llevar a la práctica, este enfoque integral y alternativo, pero todavía abierto y en construcción, asumiendo los riesgos y las potencialidades inherentes al mismo.

21 En el documento que expone de manera más extensa las ideas presentadas en este artículo, hemos recogido algunas referentes en Euskal Herria, por ejemplo, las políticas desarrolladas por los municipios de Aramaio, Orduña, Zeberio y Zerain, y las comarcas de Sakana y Urola-Garaia. 
2. Aceptar cierto grado de incertidumbre y entender que no hay recetas cerradas ni caminos trazados. No obstante, sí contamos con propuestas y experiencias que pueden servirnos como inspiración para construir alternativas adecuadas a nuestros contextos y realidades.

3. Desarrollar la creatividad. Frente a los márgenes muchas veces estrechos de las competencias, de las limitaciones presupuestarias, de las normativas, de las formas habituales de hacer, etc., necesitamos ir construyendo estas propuestas desde otros parámetros y miradas, abriendo otras ventanas, y asumiendo que el camino es en sí mismo parte del proceso de aprendizaje.

4. Replantearnos integralmente lo que entendemos por participación y construcción colectiva, revisando las formas actuales, delegadas y de bajo perfil, hacia otras políticas construidas desde otras metodologías, herramientas y espacios. No solamente es importante el resultado o los logros, los qué, sino que resultan igual de importantes los procesos, los cómos, y los entre quiénes. Por tanto, resulta necesaria la construcción de procesos en los cuales el conocimiento no quede en manos de personas consideradas expertas, sino que sea construido colectivamente y compartido, y en los cuales se garantice la participación de una mayoría amplia y diversa, garantizando siempre una presencia activa de los y las baserritarras.

5. Mantener una visión holística, integral y transversal en la definición de los planes y propuestas a nivel local, frente a la sectorialización y división de los temas, cuestión que consideramos resulta bastante habitual en el ámbito de las políticas públicas. Los retos a los que nos enfrentamos son grandes, no podemos abordar esta complejidad desde miradas simples o parciales. Por lo tanto resulta necesario sumar y garantizar que la soberanía alimentaria sea un enfoque presente y transversal en todas las políticas. Por otro lado, esta apuesta por la interdisciplinariedad o por la integralidad, puede favorecer cierta coherencia en las políticas, evitando la puesta en marcha de iniciativas que estén produciendo efectos contradictorios en la consecución del modelo alternativo que queremos impulsar.

6. Aterrizar y concretar en cada contexto y realidad particular (tamaño, características socio-demográficas, carácter rural o urbano, etc.) los enfoques de soberanía alimentaria e intersección de soberanías. Nos parece importante señalar tres ideas que consideramos debieran ser comunes: contar con enfoques de referencia que nos orienten políticamente; lanzar una mirada amplia e integral a nuestros territorios; y en tercer lugar, no olvidar que en la propuesta de la soberanía alimentaria tiene un papel central la alimentación. Es decir, para esta propuesta es central aumentar, potenciar y proteger al campesinado y a la producción de alimentos en nuestros territorios, garantizando su acceso para toda la 
población. Sin esto difícilmente podremos avanzar en la alimentación como derecho fundamental.

7. Convertir a la administración pública, en este caso local, en referente en la materia, por lo que no se trata solo de proponer acciones hacia fuera, sino que se debe también trabajar mucho hacia el interior de las propias instituciones. Así, el papel de los ayuntamientos es múltiple, como promotores, facilitadores y dinamizadores de estas transformaciones, y como agentes directamente implicados en las mismas, en su rol de productores o consumidores de diferentes bienes o servicios.

8. Reconocer la importancia de las iniciativas impulsadas por la sociedad civil, por las organizaciones o movimientos, muchas veces a pesar de las administraciones públicas, entendiendo que son proyectos de los cuales podemos extraer aprendizajes y que podrían ser facilitados y fortalecidos por una administración pública con carácter transformador.

9. Articular la realidad local con otros niveles o ámbitos. Para la realidad, tamaño y forma de organización administrativa de Euskal Herria, resulta fundamental incluir una perspectiva comarcal, en sentido amplio. La alianza, complementariedad y trabajo conjunto entre municipios nos parece una perspectiva muy interesante y necesaria para abordar, de la mejor manera posible, las profundas transformaciones que supone el paradigma de la soberanía alimentaria.

10. Asumir que nos enfrentamos a procesos a largo plazo y que para desarrollarlos deberemos dotarnos de múltiples herramientas, entre ellas el análisis y la planificación, y un seguimiento y valoración continuos de los logros y dificultades. Y esta identidad del largo plazo se enfrenta abiertamente a los ritmos y dinámicas electorales. La participación en la construcción de estos proyectos y la apropiación de los mismos por parte de la ciudadanía y de los y las baserritarras, es una condición como decíamos sine qua non para que puedan desarrollarse, pero también se convierte en una garantía de su continuidad, los convierte en menos vulnerables y dependientes, y con bases firmes para ser defendidos por todos y todas los/las que en ellos toman parte.

Nos gustaría finalizar este apartado, siguiendo con una de las ideas planteadas en este décimo principio, presentando algunas de las posibles herramientas que las políticas públicas locales nos ofrecen y que nos pueden permitir impulsar la soberanía alimentaria en nuestros territorios. Así podemos:

- Promover la construcción colectiva y acompañamiento de los procesos de soberanía alimentaria y definir los recursos necesarios para garantizar su sostenibilidad. Hacemos referencia a todo lo que tiene que ver la definición de los cómos y los quiénes, de los caminos que van a garantizar la construcción participada de las propuestas, desde la identificación de ac- 
tores relevantes; la creación de espacios para la participación; la definición y concreción de estos procesos; el establecimiento de herramientas de formación para la participación; la gestión de la información y/o la comunicación; y el establecimiento de mecanismos de seguimiento y auditoría ciudadana. Además, resulta fundamental dotarnos de los recursos necesarios para garantizar la sostenibilidad de los procesos, recursos entendidos en un sentido amplio, haciendo referencia desde luego a los presupuestos y al personal, pero también a cuestiones como el intercambio de conocimientos entre diferentes agentes y/o la generación de articulaciones y redes, entre otras.

- Ponernos las gafas de la soberanía alimentaria y conocer y analizar nuestra realidad. Esta herramienta tiene que ver con la elaboración de diagnósticos, estudios, análisis, ampliar la información, los datos y el conocimiento que tenemos de las diferentes cuestiones que se proponen abordar en los cinco ámbitos de soberanía. Ampliar la mirada y analizar la realidad es una cuestión sin la cual no podremos abordar procesos que busquen transformarla.

- Construir nuestra propuesta y establecer los pasos que queremos ir dando para desarrollarla. Esta herramienta recogería todos los procesos de planificación y elaboración de proyectos, de carácter integral o más sectorial. Se trata de establecer los objetivos, las líneas específicas de actuación, los cronogramas, los espacios y los recursos necesarios para implementarlos.

- Establecer nuevos marcos normativos que nos permitan ir poniendo en marcha estas propuestas alternativas. Esta herramienta hace referencia al desarrollo de diferentes normativas, decretos, ordenanzas, declaraciones institucionales... que puedan ir sentando las bases y ampliando los espacios posibles para el aterrizaje y el desarrollo de la agenda de la soberanía alimentaria y de la intersección de soberanías propuesta.

- Ir poniendo en práctica las ideas a través del desarrollo de proyectos concretos. En esta herramienta hacemos referencia a esas iniciativas, que nos permiten ir avanzando en el logro de los objetivos definidos en cada ámbito de soberanía. Serían iniciativas tractoras o palanca de cambio, que pueden orientar transformaciones más generales.

\section{Conclusiones}

El objetivo de este artículo era mostrar cómo la agenda de la soberanía alimentaria confronta, a múltiples niveles, las lógicas y agendas del poder corporativo. Y, en segundo lugar, proponer un marco que nos pueda servir de guía para la definición de otros modelos de vida desde lo local que respondan a esta agenda, presentando los principios y herramientas que, desde las políticas públi- 
cas locales, nos pueden ayudar a desarrollarla. No hay recetas cerradas, pero sí caminos que se están definiendo junto con otras y otros.

En el documento que ha servido como referencia para el presente artículo, se han intentado recuperar diferentes actuaciones que se están desarrollando (o se podrían) desarrollar en los cinco ámbitos de soberanía presentados (bienes naturales, energía, sistemas agroalimentarios locales, autonomía de las mujeres, lo común y el conocimiento libre). Actuaciones que si bien en ocasiones pueden ser tachadas como pequeñas o con un impacto limitado, consideramos importantes porque nos permiten no quedarnos en las reflexiones más discursivas, sino a través en muchas ocasiones del ensayo/error, poner en marcha toda esta propuesta en nuestras realidades. Muchas de ellas además están mostrando una gran capacidad de resistencia, frente a un contexto cada vez más desfavorable para ese recuperar y defender espacios de vida frente al capital.

Así, por ejemplo y por destacar algunas, existen distintas experiencias en torno a la tierra, desde la generación de bancos de tierras — con una menor o mayor participación de la administración, y que buscan fundamentalmente disponer de tierra para la producción de alimentos, intentando sacar esta de las lógicas especulativas - hasta experiencias de compra colectiva; proyectos que han apoyado la incorporación de los y las jóvenes a la producción de alimentos, en claves agroecológicas; iniciativas centradas en recuperar semillas tradicionales y generar viveros con estas variedades autóctonas; proyectos, que desde la perspectiva de la soberanía energética, han impulsado fuentes energéticas alternativas, por ejemplo la biomasa; la creación de cooperativas de generación y comercialización de energía, y la colaboración de diferentes municipios con las mismas; dinamización de mercados locales y grupos de consumo, que ponen en relación directa a las personas productoras y consumidoras; diferentes experiencias que enfrentan la actual normativa sobre comedores escolares existente en la CAE, y que están peleando frente a las grandes empresas de catering por una alimentación en las escuelas sostenible, cercana y saludable; la generación de redes entre mujeres campesinas y urbanas; la puesta en marcha de diferentes procesos formativos, impulsados tanto desde los sindicatos como desde ámbitos más académicos, con apoyo de algunas instituciones, o la promoción de mercados sociales, para conocer y reflexionar sobre propuestas de consumo alternativas.

Por lo tanto, hay propuesta, hay alternativas. Para seguir aumentando el número y profundizando el carácter transformador de las mismas, rescatamos dos ideas que consideramos centrales: proyecto colectivo y alianza. Estamos en un momento en el que la construcción de alianzas y el contagio de agendas alternativas es algo prioritario y urgente. Encontrarnos entre aquellas agendas y sujetos que están defendiendo frente a los mercados capitalistas y sus actores hegemónicos, otras alternativas que nos permitan avanzar en colocar en el centro la sostenibilidad de la vida en sentido amplio. Y dotar de contenido a la palabra alianza, 
entre actores diversos (movimientos sociales, poderes públicos con vocación transformadora, ONG, universidad, etc.), que exista una responsabilidad política compartida de todos ellos, y que cada quien desde su posición, definiendo objetivos comunes y tareas específicas, entienda como propios tanto los avances como los retrocesos. Y desde luego entendiendo que las políticas públicas deben ser siempre una herramienta para enfrentar el poder corporativo, y no un ámbito que permita su crecimiento y sostenga su impunidad. Esperamos que alguna de las ideas aportadas en este artículo nos permita avanzar en este camino.

\section{Bibliografía}

ALTIERI, Miguel y NICHOLLS, Clara, (2000), Agroecología. Teoría y práctica para una agricultura sustentable, PNUMA, en: http://www.ambiente.gov.ar/infotecaea/ descargas/altieri01.pdf

AMIGOS DE LA TIERRA (2012), «Informe Alimentos kilométricos», en: http://www. tierra.org/spip/spip.php?article1549

ARRIOLA, Iratxe; ITURBE, Ainhoa y GÓMEZ, Henar (2009), «La mujer baserritarra, esa valiosa especie en peligro de extinción", en Las mujeres alimentan el mundo. Soberanía alimentaria en defensa de la vida y el planeta, Entrepueblos, en: http://www. entrepueblos.org/files/Libro_sa_genero.pdf

BERMEJO, Isabel (2010), "Agricultura y cambio climático», Revista El Ecologista n. ${ }^{\circ} 67$, en: http://www.ecologistasenaccion.es/article19945.html

CARO, Pamela (2013), «Soberanía alimentaria: aproximaciones a un debate sobre alternativas de desarrollo y derechos de las mujeres», en Libro abierto de la Via Campesina, celebrando 20 años de luchas y esperanza, en: http://viacampesina.org/downloads/pdf/ openbooks/ES-05.pdf

ETC Group (2013), El carro delante del caballo. Semillas, suelos y campesinos. ¿Quién controla los insumos agricolas?, en: http://www.etcgroup.org/es/content/el-carro-delantedelcaballo-semillas-suelos-y-campesinos

ETXALDE (2015), TTIP-TAFTAren aurrean Nekazaritza iraunkorra eta elikadura burujabetzaren garaia da, en:https://drive.google.com/file/d/0B3nyLyf2hTxiSG56WmZKWGNZc2c/ view?pli $=1$

FERNÁNDEZ, Gonzalo (2012), «Soberanía alimentaria», en Hernández Zubizarreta, Juan, González, Erika y Ramiro, Pedro, Diccionario crítico de empresas transnacionales. Claves para enfrentar el poder de las grandes corporaciones, en:http://omal.info/spip. php?rubrique31

FERNANDEZ, Gonzalo; PIRIS, Silvia y RAMIRO, Pedro (2013), Cooperación internacional y movimientos sociales emancipadores. Bases para un encuentro necesario, Hegoa, Bilbao. Documento disponible en: http://biblioteca2012.hegoa.efaber.net/registros/19540

FERNÁNDEZ, Gonzalo (2015), Alternativas para disputar el poder, el ser y el saber a las transnacionales, Revista Pueblos, n. ${ }^{\circ}$ 66. Disponible en: http://omal.info/spip. php?article7179

GALLAR, David (2013), "Economías campesinas como cultura a rescatar», en Revista Soberania alimentaria. Biodiversidad y culturas, n. ${ }^{\circ}$ 12, julio de 2013, en: http://www. soberaniaalimentaria.info/publicados/numero-12/31-portada-n12 
GARCÍA, Estefanía (2012), «Ecofeminismos rurales. Mujeres por la soberanía alimentaria», Mundubat y Revista Soberania alimentaria, biodiversidad y culturas, en: https:// redecofeminista.files.wordpress.com/2012/11/ecofem_rurales_web.pdf

GRAIN (2009a), Los nuevos dueños de la tierra, en: https:/www.grain.org/article/ entries/195-los-nuevos-duenos-de-la-tierra

GRAIN (2009b), La agricultura, sus saberes y cuidados, en: https://www.grain.org/fr/ article/entries/1201-la-agriculturasus-saberes-y-cuidados

HERNÁNDEZ ZUBIZARRETA, Juan, GONZÁLEZ, Erika y RAMIRO, Pedro (2012), Diccionario crítico de empresas transnacionales. Claves para enfrentar el poder de las grandes corporaciones, versión electrónica en:http://omal.info/spip.php?rubrique31

HERRERO, Yayo (2015), "Apuntes introductorios sobre el ecofeminismo», en: http:// publicaciones.hegoa.ehu.es/assets/pdfs/334/Boletin_n\%C2\%BA43.pdf?1437989000

LA VÍA CAMPESINA (2009), Documentos politicos, 5. a Conferencia de Mozambique, en:http://viacampesina.org/es/index.php/publicaciones-mainmenu-30/943ocumentospoliticoslvc

LA VÍA CAMPESINA (2013), "Nuestras semillas, nuestro futuro», Cuadernos de La Vía Campesina, disponible en: http://viacampesina.org/downloads/pdf/sp/ES-notebook6.pdf

LA VIA CAMPESINA, AMIGOS DE LA TIERRA INTERNACIONAL Y COMBAT MONSANTO (2012), Lucha contra Monsanto. Resistencia de los movimientos de base al poder empresarial del agronegocio en la era de la economía verde y un clima cambiante, en: http://www.viacampesina.org/downloads/pdf/sp/Monsanto-Publication-ES-FinalVersion.pdf

LÓPEZ, Daniel y LLORENTE, Mireia (2010), «La agroecología: hacia un nuevo modelo agrario Sistema agroalimentario, producción ecológica y consumo responsable», Ecologistas en Acción, en: http://www.ecologistasenaccion.org/IMG/pdf_cuaderno_17_ agroecologia.pdf

PÉREZ OROZCO, Amaia (2012), De vidas vivibles y producciones imposibles, en: http:// www.decrecimiento.info/2012/02/de-vidas-vivibles-y-produccion.html

ROSSET, Peter y MARTÍNEZ TORRES, María Elena (2013), «La Vía Campesina y Agroecología", en Libro abierto de la Vía Campesina, celebrando 20 años de luchas y esperanza, enhttp://viacampesina.org/downloads/pdf/openbooks/ES-09.pdf

SEVILLA, Eduardo (2006), Agroecología y soberanía alimentaria: alternativas a la globalización agroalimentaria, en: http://institucional.us.es/compromiso/libreconf/docs/ agroecologia.pdf

TNI: Transnational Institute y Fuhem Ecosocial (2013), El acaparamiento global de tierras. Guía básica, en: https://www.fuhem.es/media/cdv/file/biblioteca/Analisis/2013/ El-acaparamiento-de-tierras_Guia-basica_junio2013.pdf

VSF: Justicia Alimentaria Global, Cortocircuito. Por una alimentación sana, cercana y justa (campaña, investigación y guía práctica para las administraciones locales), en:http:// vsf.org.es/cortocircuito

ZACUNE, Joseph (2013), Privatizando Europa. La crisis como tapadera para consolidar el neoliberalismo, TNI-Transnational Institute, en: http://www.tni.org/sites/www.tni. org/files/download/privatising_europe-es.pdf

ZUBIALDE, Xabier (2014), Guía hacia la soberania energética de Euskal Herria. 13 líneas estratégicas a nivel global. 13 pasos a dar a nivel local, en:http://www4.gipuzkoa. net/MedioAmbiente/gipuzkoaingurumena/adj/documentacion/Guia\%20hacia $\% 20$ la20soberan\%C3\%ADa\%20energ\%C3\%A9tica\%20de\%20Euskal\%20Herria.pdf 